\title{
Colchicine in patients admitted to hospital with COVID-19 (RECOVERY): a randomised, controlled, open-label, platform trial
}

RECOVERY Collaborative Group*

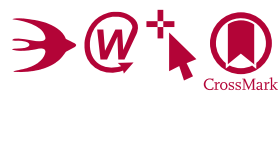

\section{Summary}

Background Colchicine has been proposed as a treatment for COVID-19 based on its anti-inflammatory actions. We aimed to evaluate the efficacy and safety of colchicine in patients admitted to hospital with COVID-19.

Methods In this streamlined, randomised, controlled, open-label trial, underway at 177 hospitals in the UK, two hospitals in Indonesia, and two hospitals in Nepal, several possible treatments were compared with usual care in patients hospitalised with COVID-19. Patients were eligible for inclusion in the study if they were admitted to hospital with clinically suspected or laboratory confirmed SARS-CoV-2 infection and had no medical history that might, in the opinion of the attending clinician, put the patient at significant risk if they were to participate in the trial. Eligible and consenting adults were randomly assigned (1:1) to receive either usual standard of care alone (usual care group) or usual standard of care plus colchicine (colchicine group) using web-based simple (unstratified) randomisation with allocation concealment. Participants received colchicine $1 \mathrm{mg}$ after randomisation followed by $500 \mu \mathrm{gg}$ later and then $500 \mu \mathrm{g}$ twice a day by mouth or nasogastric tube for 10 days in total or until discharge. Dose frequency was halved for patients receiving a moderate CYP3A4 inhibitor (eg, diltiazem), patients with an estimated glomerular filtration rate of less than $30 \mathrm{~mL} / \mathrm{min}$ per $1.73 \mathrm{~m}^{2}$, and those with an estimated bodyweight of less than $70 \mathrm{~kg}$. The primary outcome was 28-day mortality, secondary endpoints included time to discharge, the proportion of patients discharged from hospital within 28 days, and, in patients not on invasive mechanical ventilation at randomisation, a composite endpoint of invasive mechanical ventilation or death. All analyses were by intention-to-treat. The trial is registered with ISRCTN, 50189673, and ClinicalTrials.gov, NCT04381936.

Findings Between Nov 27, 2020, and March 4, 2021, 11340 (58\%) of 19423 patients enrolled into the RECOVERY trial were eligible to receive colchicine; 5610 (49\%) patients were randomly assigned to the colchicine group and 5730 (51\%) to the usual care group. Overall, $1173(21 \%)$ patients in the colchicine group and $1190(21 \%)$ patients in the usual care group died within 28 days (rate ratio 1.01 [95\% CI 0.93 to 1.10$]$; $\mathrm{p}=\mathbf{0} \cdot 77$ ). Consistent results were seen in all prespecified subgroups of patients. Median time to discharge alive (10 days [IQR 5 to $>28]$ ) was the same in both groups, and there was no significant difference in the proportion of patients discharged from hospital alive within 28 days (3901 [70\%] patients in the colchicine group and 4032 [70\%] usual care group; rate ratio 0.98 [95\% CI 0.94 to 1.03 ]; $\mathrm{p}=0.44$ ). In those not on invasive mechanical ventilation at baseline, there was no significant difference in the proportion meeting the composite endpoint of invasive mechanical ventilation or death (1344 [25\%] in the colchicine group vs 1343 [25\%] patients in the usual care group; risk ratio 1.02 [95\% CI 0.96 to 1.09]; p=0 47).

Interpretation In adults hospitalised with COVID-19, colchicine was not associated with reductions in 28-day mortality, duration of hospital stay, or risk of progressing to invasive mechanical ventilation or death.

Funding UK Research and Innovation (Medical Research Council), National Institute of Health Research, and Wellcome Trust.

Copyright (C) 2021 The Author(s). Published by Elsevier Ltd. This is an Open Access article under the CC BY 4.0 license.

\section{Introduction}

Inflammation is a key feature of severe COVID-19. Markedly raised concentrations of inflammatory markers, such as C-reactive protein (CRP), ferritin, interleukin-6 (IL-6), and other cytokines, are observed in severe cases and are associated with poor outcomes. ${ }^{1-5}$ Inflammation is particularly prominent in the lung and vascular endothelium, and is commonly associated with extensive alveolar damage and thrombosis of large and small pulmonary vessels. ${ }^{6}$ Corticosteroids and IL-6 inhibitors have both been shown to reduce mortality in patients with severe COVID-19; Janus kinase (JAK) inhibitors accelerate improvement in clinical status. ${ }^{7-10}$ Together these results show that inflammation is modifiable and anti-inflammatory regimens can improve clinical outcomes.

Inflammasomes are a key part of the innate immune response to SARS-CoV-2 infection. These cytosolic pattern recognition receptor systems are activated in response to detection of pathogens in the cytosol and stimulate the
Lancet Respir Med 2021

Published Online October 18, 2021

https://doi.org/10.1016/ S2213-2600(21)00435-5

*The writing committee and trial steering committee are listed at the end of this manuscript and complete list of collaborators in the Randomised Evaluation of COVID-19 Therapy (RECOVERY) trial is provided in the appendix (pp 2-25).

Correspondence to: Prof Peter W Horby and Prof Martin J Landray, RECOVERY Central Coordinating Office, Oxford OX3 7LF, United Kingdom recoverytrial@ndph.ox.ac.uk See Online for appendix 
Research in context

\section{Evidence before this study}

We searched medRxiv, bioRxiv, Medline, Embase, and the WHO International Clinical Trials Registry Platform from Sept 1, 2019, to April 1, 2021, for clinical trials evaluating the effect of colchicine treatment in patients hospitalised with COVID-19, using the search terms ("SARS-CoV-2.mp" OR "COVID.mp" OR "COVID-19.mp" OR "2019-nCoV.mp" OR "Coronavirus.mp" OR "Coronavirinae/") AND ("colchicine.mp" OR "colchicine/"), using validated filters to select for randomised controlled trials. No language restrictions were applied.

We identified three relevant randomised trials that compared colchicine with usual care or placebo in patients hospitalised with COVID-19 (two at low risk of bias and one with some concerns because of limited information on randomisation process and poor clarity regarding the blinding in the study). Each trial suggested a potential favourable effect of colchicine on outcome measures of clinical improvement or duration of hospitalisation. The three trials combined included a total of
285 patients and seven deaths; even combined, these trials were not adequately powered to detect an effect on mortality.

\section{Added value of this study}

The RECOVERY trial is the first large, randomised trial to report results of the effect of colchicine in patients hospitalised with COVID-19. We found no significant effect of colchicine compared with usual care alone on 28-day mortality, the probability of discharge alive within 28 days, or the probability of progressing to the composite outcome of invasive mechanical ventilation or death in patients who were not receiving invasive mechanical ventilation at randomisation. We saw no evidence of benefit of colchicine in any patient subgroup.

\section{Implications of all the available evidence}

There is no good evidence that colchicine treatment is of clinical benefit for adults hospitalised with COVID-19 compared with current usual care.
For more the protocol, statistical analysis plan, and additional study resource see www. recoverytrial.net release of proinflammatory cytokines. ${ }^{11}$ In COVID-19, the degree of inflammasome activation, particularly the nucleotide binding domain (NOD)-like pyrin domain 3 (NLRP3) inflammasome, correlates with disease severity. ${ }^{12}$ Colchicine, a readily available, safe, and inexpensive drug, has a wide range of anti-inflammatory effects, including inhibition of the NLRP3 inflammasome. ${ }^{13}$ In addition to its role in treating acute gout and pericarditis, evidence is emerging that colchicine might inhibit endovascular inflammation and provide clinical benefits in patients with coronary artery disease. ${ }^{14-17}$ Given the activation of NLRP3 in COVID-19 and the presence of vascular endothelial inflammation, colchicine has been proposed as a treatment for SARS-CoV-2 associated inflammatory disease. However, only three small randomised trials have assessed the effects of colchicine in hospitalised patients with COVID-19, with a total of only seven deaths across these studies combined; none of the studies were adequately powered to identify any effect of the drug on mortality. ${ }^{18-20}$ Here we report the results of a large randomised controlled trial that aimed to evaluate the efficacy and safety of colchicine in patients hospitalised with COVID-19.

\section{Methods}

\section{Study design and participants}

The RECOVERY trial is an investigator-initiated, streamlined, individually randomised, controlled, openlabel, platform trial to evaluate the effects of potential treatments in patients hospitalised with COVID-19. Details of the trial design and results for other possible treatments (dexamethasone, ${ }^{7}$ hydroxychloroquine, ${ }^{21}$ lopinavir-ritonavir, ${ }^{22}$ azithromycin ${ }^{23}$ tocilizumab, ${ }^{2}$ and convalescent plasma ${ }^{24}$ ) have been published previously. The trial is underway at 177 hospitals in the UK, two hospitals in Indonesia, and two hospitals in Nepal (appendix pp 3-25). The trial is supported by the National Institute for Health Research Clinical Research Network, and is coordinated by the Nuffield Department of Population Health (University of Oxford, Oxford, UK), the trial sponsor. The trial was done in accordance with the principles of the International Conference on Harmonisation-Good Clinical Practice guidelines and approved by the UK Medicines and Healthcare products Regulatory Agency (MHRA) and the Cambridge East Research Ethics Committee (20/EE/0101). The protocol, statistical analysis plan, and additional information are available online.

Patients admitted to hospital were eligible for the study if they had clinically suspected or laboratory confirmed SARS-CoV-2 infection and no medical history that might, in the opinion of the attending clinician, put the patient at significant risk if they were to participate in the trial. Children and pregnant women were not eligible to receive colchicine. Patients with severe liver impairment, significant cytopaenia concomitant use of strong CYP3A4 (eg, clarithromycin, erythromycin, systemic azole antifungal, and HIV protease inhibitor) or P-glycoprotein inhibitors (eg, ciclosporin, verapamil, and quinidine), or hypersensitivity to lactose were excluded from the colchicine comparison (appendix p 81). Written informed consent was obtained from all patients, or a legal representative if patients were too unwell or unable to provide consent.

\section{Randomisation and masking}

Baseline data were collected using a web-based case report form that included demographics, level of respiratory support, major comorbidities, suitability of the study treatment for a particular patient, and treatment 
availability at the study site (appendix pp 32-34). Eligible and consenting, non-pregnant adult patients were randomly assigned (1:1) to receive either usual standard of care (usual care group) or usual standard of care plus colchicine (colchicine group), or one of the other available RECOVERY treatment groups, using web-based simple (unstratified) randomisation with allocation concealed until after randomisation (appendix pp 30-31). For some patients, colchicine was unavailable at the hospital at the time of enrolment or was considered by the managing physician to be either definitely indicated or definitely contraindicated. These patients were excluded from the randomised comparison between colchicine and usual care. Patients received colchicine $1 \mathrm{mg}$ after randomisation followed by $500 \mu \mathrm{g} 12 \mathrm{~h}$ later and then $500 \mu \mathrm{g}$ twice a day orally or by nasogastric tube for 10 days in total or until discharge, whichever occurred first. Dose frequency was halved for patients receiving a moderate CYP3A4 inhibitor (eg, diltiazem), those who had renal impairment (estimated glomerular filtration rate $<30 \mathrm{~mL} / \mathrm{min}$ per $1.73 \mathrm{~m}^{2}$ ), and patients with an estimated bodyweight of less than $70 \mathrm{~kg}$ (appendix p 81).

As a platform trial, and in a factorial design, patients could be simultaneously randomly assigned to other treatment groups: 1) convalescent plasma versus casirivimab and imdevimab versus usual care, 2) aspirin versus usual care, and 3) baricitinib versus usual care (appendix p 31). Until Jan 24, 2021, the trial also allowed a subsequent randomisation for patients with progressive COVID-19 (evidence of hypoxia and a hyperinflammatory state) to receive usual care plus tocilizumab or usual care alone. Participants and local study staff were not masked to the allocated treatment. The trial steering committee, investigators, and all other individuals involved in the trial were masked to outcome data during the trial.

\section{Procedures}

A single online follow-up form was completed when participants were discharged, had died, or 28 days after randomisation, whichever occurred first (appendix pp 35-41). Information was recorded on adherence to allocated study treatment, receipt of other COVID-19 treatments, duration of admission, receipt of respiratory or renal support, and vital status (including cause of death). In addition, in the UK, routine health-care and registry data were obtained, including information on vital status (with date and cause of death), discharge from hospital, receipt of respiratory support, or renal replacement therapy.

\section{Outcomes}

Outcomes were assessed 28 days after randomisation, with additional analyses specified at 6 months. The primary outcome was all-cause mortality. Secondary outcomes were time to discharge from hospital alive within 28 days and, in patients not on invasive mechanical ventilation at randomisation, receipt of invasive mechanical ventilation (including extracorporal membrane oxygenation) or death. Prespecified subsidiary clinical outcomes were use of noninvasive respiratory support, time to successful cessation of invasive mechanical ventilation (defined as cessation of invasive mechanical ventilation within, and survival to, 28 days), use of haemodialysis or haemofiltration, causespecific mortality, bleeding events, thrombotic events, and major cardiac arrhythmias. Information on suspected serious adverse reactions was collected in an expedited fashion to comply with regulatory requirements.

\section{Statistical analysis}

The primary analysis for all outcomes was assessed according to the intention-to-treat principle by comparing patients randomly assigned to the colchicine group with those who were randomly assigned to the usual care group, but for whom colchicine was both available and a suitable treatment. For the primary outcome, all-cause 28-day mortality, the log-rank observed minus expected statistic and its variance were used to test both the null hypothesis of equal survival curves (ie, the log-rank test) and to calculate the one-step estimate of the average mortality rate ratio. We used Kaplan-Meier survival curves to display cumulative mortality over the 28-day period. We used the same method to analyse time to hospital discharge and successful cessation of invasive

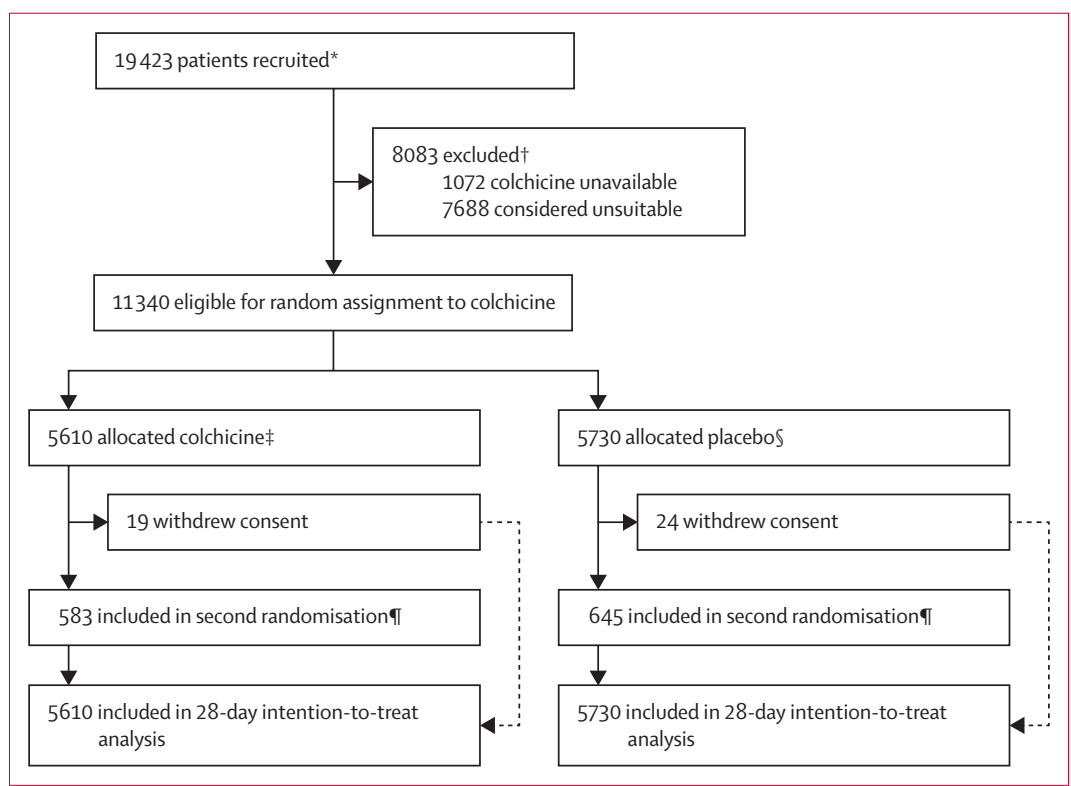

Figure 1: Trial profile

Of the 11340 patients randomly assigned to receive colchicine or usual care, 7091 (63\%; 3505 [62\%] of the colchicine group vs 3586 [63\%] of the usual care group) patients were additionally randomised to receive convalescent plasma or REGN-COV2 or usual care; 7545 (67\%; 3747 [67\%] of the colchicine group vs 3798 [66\%] of the usual care group) patients were additionally randomised to receive aspirin or usual care; and 1635 (14\%; 802 [14\%] of the colchicine group vs 833 [15\%] of the usual care group) patients were additionally randomised to receive baricitinib or usual care. *Number recruited overall during period that adult participants could be recruited into colchicine comparison. †Colchicine unavailable and colchicine unsuitable groups are not mutually exclusive. $\$ 5122$ (93\%) of 5510 patients with completed follow-up at time of analysis received colchicine. $\$ 20(<1 \%)$ of 5605 patients with completed follow-up at time of analysis received colchicine. IIncludes 251 (4\%) of 5610 patients in the colchicine group and $306(5 \%)$ of 5730 patients in the usual care group allocated to receive tocilizumab. 


\begin{tabular}{|c|c|c|}
\hline & $\begin{array}{l}\text { Colchicine group } \\
(n=5610)\end{array}$ & $\begin{array}{l}\text { Usual care group } \\
(n=5730)\end{array}$ \\
\hline Age, years & $63 \cdot 3(13 \cdot 8)$ & $63.5(13 \cdot 7)$ \\
\hline \multicolumn{3}{|l|}{ Age groups, years } \\
\hline$<70$ & $3806(68 \%)$ & $3850(67 \%)$ \\
\hline 70 to $<80$ & $1139(20 \%)$ & $1227(21 \%)$ \\
\hline$\geq 80$ & $665(12 \%)$ & $653(11 \%)$ \\
\hline \multicolumn{3}{|l|}{ Sex } \\
\hline Male & $3897(69 \%)$ & $4012(70 \%)$ \\
\hline Female & $1713(31 \%)$ & $1718(30 \%)$ \\
\hline \multicolumn{3}{|l|}{ Ethnicity } \\
\hline White & $4344(77 \%)$ & $4383(76 \%)$ \\
\hline $\begin{array}{l}\text { Black, Asian, and } \\
\text { minority ethnic }\end{array}$ & $758(14 \%)$ & $813(14 \%)$ \\
\hline Unknown & $508(9 \%)$ & $534(9 \%)$ \\
\hline $\begin{array}{l}\text { Number of days since } \\
\text { symptom onset }\end{array}$ & $9(6-12)$ & $9(6-12)$ \\
\hline $\begin{array}{l}\text { Number of days since } \\
\text { admission to hospital }\end{array}$ & $2(1-3)$ & $2(1-3)$ \\
\hline \multicolumn{3}{|l|}{ Respiratory support received } \\
\hline None or simple oxygen & $3815(68 \%)$ & $3962(69 \%)$ \\
\hline $\begin{array}{l}\text { Non-invasive } \\
\text { ventilation }\end{array}$ & $1527(27 \%)$ & $1507(26 \%)$ \\
\hline $\begin{array}{l}\text { Invasive mechanical } \\
\text { ventilation }\end{array}$ & $268(5 \%)$ & $261(5 \%)$ \\
\hline \multicolumn{3}{|l|}{ Laboratory measurements } \\
\hline $\begin{array}{l}\text { C-reactive protein, } \\
\mathrm{mg} / \mathrm{L}\end{array}$ & $86(44-145)$ & $87(46-144)$ \\
\hline Creatinine, $\mu \mathrm{mol} / \mathrm{L}$ & $78(64-96)$ & $78(65-96)$ \\
\hline \multicolumn{3}{|l|}{ Previous diseases } \\
\hline Diabetes & $1426(25 \%)$ & $1470(26 \%)$ \\
\hline Heart disease & $1189(21 \%)$ & $1231(21 \%)$ \\
\hline Chronic lung disease & $1208(22 \%)$ & $1206(21 \%)$ \\
\hline Tuberculosis & $16(<1 \%)$ & $13(<1 \%)$ \\
\hline HIV & $11(<1 \%)$ & $20(<1 \%)$ \\
\hline Severe liver disease* & 0 & 0 \\
\hline $\begin{array}{l}\text { Severe kidney } \\
\text { impairment } \dagger\end{array}$ & $170(3 \%)$ & $166(3 \%)$ \\
\hline Any of the above & $2880(51 \%)$ & $2963(52 \%)$ \\
\hline \multicolumn{3}{|l|}{ Use of corticosteroids } \\
\hline Yes & $5243(93 \%)$ & $5360(94 \%)$ \\
\hline No & $363(6 \%)$ & $365(6 \%)$ \\
\hline Missing & $4(<1 \%)$ & $5(<1 \%)$ \\
\hline Use of remdesivir & $1235(22 \%)$ & $1251(22 \%)$ \\
\hline \multicolumn{3}{|l|}{ SARS-CoV-2 PCR test result } \\
\hline Positive & $5456(97 \%)$ & $5553(97 \%)$ \\
\hline Negative & $57(1 \%)$ & $58(1 \%)$ \\
\hline Unknown & $97(2 \%)$ & $119(2 \%)$ \\
\hline
\end{tabular}

Data are mean (SD), $\mathrm{n}(\%)$, or median (IQR). No children or pregnant women were randomised. *Defined as requiring ongoing specialist care. †Defined as estimated glomerular filtration rate less than $30 \mathrm{~mL} / \mathrm{min}$ per $1.73 \mathrm{~m}^{2}$.

Table 1: Baseline characteristics

mechanical ventilation, with patients who died in hospital right-censored on day 29. Median time to discharge was derived from Kaplan-Meier estimates. For

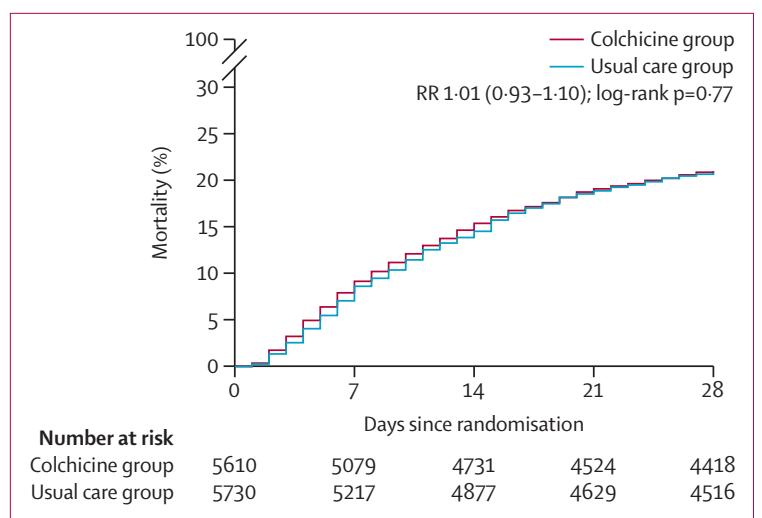

Figure 2: Effect of allocation to colchicine on 28-day mortality

the prespecified composite secondary outcome of progression to invasive mechanical ventilation or death within 28 days in those not receiving invasive mechanical ventilation at randomisation, and the subsidiary clinical outcomes of receipt of ventilation and use of haemodialysis or haemofiltration, the precise dates were not available and so the risk ratio was estimated.

Prespecified analyses were done for the primary outcome using the statistical test of interaction (test for heterogeneity or trend), in accordance with the prespecified analysis plan, defined by characteristics at randomisation: age, sex, ethnicity, level of respiratory support, days since symptom onset, and use of corticosteroids (appendix p 115). An exploratory analysis of the primary outcome by CRP concentration at randomisation was done using a similar approach.

Estimates of rate ratios and risk ratios are shown with 95\% CIs. All $\mathrm{p}$ values are two-sided and are shown without adjustment for multiple testing. The full database is held by the study team, which collected the data from study sites and did the analyses at the Nuffield Department of Population Health, University of Oxford (Oxford, UK).

As stated in the protocol, appropriate sample sizes could not be estimated when the trial was being planned at the start of the COVID-19 pandemic (appendix p 55). As the trial progressed, the trial steering committee, whose members were unaware of the results of the trial comparisons, determined that sufficient patients should be enrolled to provide at least $90 \%$ power at a two-sided significance level of 0.01 to detect a clinically relevant proportional reduction in 28-day mortality of $12.5 \%$ between the two groups.

On March 4, 2021, the independent data monitoring committee did a routine review of the available safety and efficacy data. The independent data monitoring committee notified the chief investigators that there was no convincing evidence that continued recruitment to the colchicine comparison would provide conclusive proof of worthwhile mortality benefit either overall or in any prespecified subgroup. Consequently, recruitment to the 


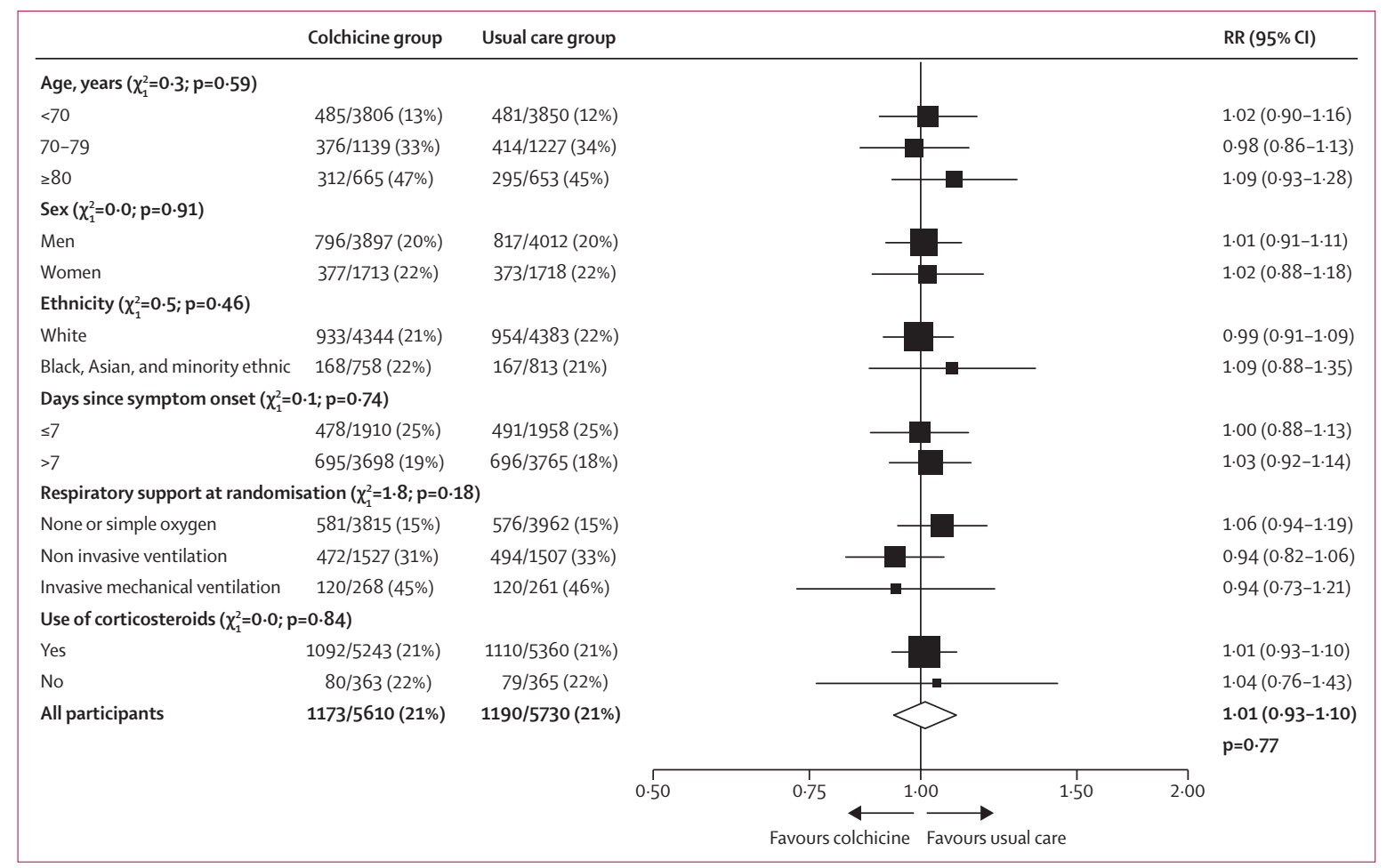

Figure 3: Effect of allocation to colchicine on 28-day mortality by baseline characteristics

Ethnicity, days since onset, and use of corticosteroids subgroups exclude those with missing data, but these patients are included in the overall summary.

colchicine comparison was closed on March 5, 2021, and preliminary results were made available to the public.

Analyses were done using SAS (version 9.4) and ( $R$ version 3.4). The trial is registered with ISRCTN, 50189673, and ClinicalTrials.gov, NCT04381936.

\section{Role of the funding source}

The funder of the study had no role in study design, data collection, data analysis, data interpretation, or writing of the report. The corresponding authors had full access to all the data in the study and had final responsibility for the decision to submit for publication.

\section{Results}

Between Nov 27, 2020, and March 4, 2021, 11340 (58\%) of 19423 patients enrolled into the RECOVERY trial were eligible to receive colchicine (ie, colchicine was available in the hospital at the time of their admission and the attending clinician was of the opinion that the patient had no known indication for or contraindication to colchicine; figure 1). 5610 (49\%) patients were randomly assigned to the colchicine group and 5730 (51\%) were randomly assigned to the usual care group $(36[<1 \%]$ patients were randomly assigned in Nepal and Indonesia). The mean age of study participants was 63.4 years (SD 13.8), and the median time since symptom onset was 9 days (IQR 6-12; table 1; appendix p 44) in both groups. At randomisation, 10603 (94\%) of patients were receiving corticosteroids.
The follow-up form was completed for 5510 (98\%) patients in the colchicine group and 5605 (98\%) patients in the usual care group. Of the patients with a completed follow-up form, 5122 (93\%) in the colchicine group received at least one dose of colchicine. Of those assigned to the colchicine group who received at least one dose, 3823 (75\%) received all (or nearly all, missing at most 1 day of treatment) of their scheduled doses during their hospital stay; $4576(90 \%)$ received at least half of their scheduled doses (figure 1; appendix p 45). The median duration of treatment with colchicine was 6 days (IQR 3-9). Use of other treatments for COVID-19 was similar between patients in both groups, with 9675 (87\%) receiving a corticosteroid, 2542 (23\%) receiving remdesivir, and 1485 (13\%) receiving tocilizumab or sarilumab (appendix p 45).

Primary outcome data are known for 11282 (99\%) of the randomly assigned patients. There was no significant difference in the proportion of patients who died within 28-days between the two groups (1173 [21\%] patients in the colchicine group vs 1190 [21\%] patients in the usual care group; rate ratio 1.01 [95\% CI $0 \cdot 93-1 \cdot 10]$; $\mathrm{p}=0 \cdot 77$; figure 2). We observed similar results across all prespecified subgroups (figure 3) and in an exploratory analysis by baseline CRP concentration (appendix p 49). In an exploratory analysis restricted to the 11009 (97\%) patients with a positive SARS-CoV-2 test result, the result was virtually identical to the overall result (rate ratio 1.02 $[0 \cdot 94-1 \cdot 10] ; \mathrm{p}=0 \cdot 70)$. 


\begin{tabular}{|c|c|c|c|c|}
\hline & $\begin{array}{l}\text { Colchicine group } \\
(n=5610)\end{array}$ & $\begin{array}{l}\text { Usual care group } \\
(n=5730)\end{array}$ & $\operatorname{RR}(95 \% \mathrm{Cl})$ & p value \\
\hline \multicolumn{5}{|l|}{ Primary outcome } \\
\hline 28-day mortality & $1173(21 \%)$ & $1190(21 \%)$ & $1.01(0.93-1 \cdot 10)$ & $0 \cdot 77$ \\
\hline \multicolumn{5}{|l|}{ Secondary outcomes } \\
\hline $\begin{array}{l}\text { Median time to being discharged } \\
\text { alive, days }\end{array}$ & $10(5$ to $>28)$ & $10(5$ to $>28)$ &. &. \\
\hline $\begin{array}{l}\text { Discharged from hospital within } \\
28 \text { days }\end{array}$ & $3901(70 \%)$ & $4032(70 \%)$ & $0.98(0.94-1.03)$ & 0.44 \\
\hline $\begin{array}{l}\text { Receipt of invasive mechanical } \\
\text { ventilation or death* }\end{array}$ & $1344 / 5342(25 \%)$ & $1343 / 5469(25 \%)$ & $1.02(0.96-1.09)$ & 0.47 \\
\hline Invasive mechanical ventilation & $600 / 5342(11 \%)$ & $591 / 5469(11 \%)$ & $1.04(0.93-1.16)$ & 0.48 \\
\hline Death & $1053 / 5342(20 \%)$ & $1070 / 5469(20 \%)$ & $1.01(0.93-1.09)$ & 0.85 \\
\hline \multicolumn{5}{|c|}{ Prespecified subsidiary clinical outcomes } \\
\hline Receipt of ventilation $\dagger$ & $852 / 3815(22 \%)$ & $941 / 3962(24 \%)$ & $0.94(0.87-1.02)$ & $0 \cdot 14$ \\
\hline Non-invasive ventilation & $818 / 3815(21 \%)$ & $904 / 3962(23 \%)$ & $0.94(0.86-1.02)$ & 0.14 \\
\hline Invasive mechanical ventilation & $259 / 3815(7 \%)$ & $228 / 3962(6 \%)$ & $1.18(0.99-1.40)$ & 0.06 \\
\hline $\begin{array}{l}\text { Successful cessation of invasive } \\
\text { mechanical ventilation } \neq\end{array}$ & $88 / 268(33 \%)$ & $81 / 261(31 \%)$ & $1 \cdot 01(0 \cdot 75-1 \cdot 37)$ & 0.93 \\
\hline $\begin{array}{l}\text { Use of haemodialysis or } \\
\text { haemofiltration } \$\end{array}$ & $212 / 5570(4 \%)$ & $203 / 5683(4 \%)$ & $1.07(0.88-1.29)$ & 0.51 \\
\hline \multicolumn{5}{|c|}{$\begin{array}{l}\text { Data are } \mathrm{n}(\%) \text { or } \mathrm{n} / \mathrm{N}(\%) \text {. RR=rate ratio for the outcomes of } 28 \text {-day mortality, hospital discharge, and successful } \\
\text { cessation of invasive mechanical ventilation, and risk ratio for other outcomes. *Analyses exclude those on invasive } \\
\text { mechanical ventilation at randomisation. †Analyses exclude those on any form of ventilation at randomisation. } \\
\ddagger \text { Analyses restricted to those on invasive mechanical ventilation at randomisation. SAnalyses exclude those on } \\
\text { haemodialysis or haemofiltration at randomisation. }\end{array}$} \\
\hline
\end{tabular}

The median time to discharge from hospital alive was 10 days (IQR 5 to $>28$ ) in both groups, and there was no significant difference in the probability of being discharged alive within 28 days between the two groups (3901 [70\%] patients in the colchicine group and 4032 (70\%) usual care group; rate ratio 0.98 [95\% CI 0.94 to 1.03 ]; $\mathrm{p}=0.44$; table 2 ). Of the patients not on invasive mechanical ventilation at baseline, the number of patients progressing to the prespecified composite secondary outcome of invasive mechanical ventilation or death was similar in both groups (1344 [25\%] in the colchicine group vs 1343 [25\%] patients in the usual care group; risk ratio 1.02 [ $95 \%$ CI 0.96 to 1.09 ]; $\mathrm{p}=0.47$ ). Similar results were seen in all prespecified subgroups of patients (appendix pp 50-51).

We found no significant differences in the prespecified subsidiary clinical outcomes of cause-specific mortality (appendix p 46), use of ventilation, successful cessation of invasive mechanical ventilation, or need for haemodialysis or haemofiltration (table 2). The incidence of new cardiac arrhythmias, bleeding events, and thrombotic events was also similar in the two groups (appendix p 47). There were two reports of a serious adverse reaction believed related to colchicine: one patient had severe acute kidney injury and one had rhabdomyolysis.

\section{Discussion}

In this large, randomised trial involving more than 11000 patients from three countries and more than
2000 deaths, use of colchicine was not associated with a reduction in mortality, duration of hospitalisation, or the risk of being ventilated or dying for those not on ventilation at baseline. These results were consistent across the prespecified subgroups of age, sex, ethnicity, duration of symptoms before randomisation, level of respiratory support at randomisation, and use of corticosteroids.

The benefit of dexamethasone in patients with COVID-19 requiring respiratory support shows the importance of inflammation in this patient group and colchicine was proposed as a treatment for COVID-19 based on its antiinflammatory activity. ${ }^{25}$ However, in this large, well powered trial, we found no evidence of a benefit from colchicine, which suggests that the anti-inflammatory properties of colchicine are either insufficient to produce a meaningful reduction in mortality risk or are not affecting the relevant inflammatory pathways in moderate-to-severe COVID-19. The protocol included a maximum of 10 days of treatment with colchicine. It is possible that a longer duration of therapy might have provided benefit, but most participants had stopped colchicine before 10 days either because of death, discharge from hospital, or at the discretion of the treating clinician. Although, most patients in this study received concomitant corticosteroid therapy, we saw no evidence that colchicine was beneficial in those patients not receiving a corticosteroid.

Strengths of this trial included that it was randomised, had a large sample size, broad eligibility criteria, was international, and more than $99 \%$ of patients were followed up for the primary outcome of all-cause mortality. However, there are some limitations. Detailed information on laboratory markers of inflammation and immune response and information on radiological features was not collected; therefore, it is not possible to assess if the effect of treatment varied between such subgroups of patients. Although this randomised trial is open-label, the outcomes are unambiguous and were ascertained without bias through linkage to routine health records.

Three other randomised controlled trials have assessed the efficacy of colchicine for the treatment of patients hospitalised with COVID-19. ${ }^{18-20}$ A two day shorter duration of hospitalisation was reported in a trial of 100 patients with laboratory confirmed SARS-CoV-2 infection and pulmonary involvement-confirmed by CT-who were randomly assigned to receive either hydroxychloroquine plus colchicine or hydroxychloroquine plus placebo. ${ }^{18} \mathrm{~A}$ second trial reported a reduced duration of hospitalisation and oxygen therapy in 36 patients hospitalised with COVID-19 allocated colchicine compared with 36 patients allocated usual care, which included hydroxychloroquine, azithromycin, and methylprednisolone. ${ }^{19}$ Finally, the GRECCO-19 trial ${ }^{20}$ reported a lower rate of clinical deterioration in 55 patients randomly assigned to receive colchicine compared with 50 patients randomly assigned to receive usual care, which 
did not include corticosteroids. ${ }^{20}$ The total number of patients in all three of these trials combined was 285, with seven deaths during the follow-up period, meaning that, even combined, these three studies are not able to reliably assess the effects of colchicine on mortality. By contrast, the RECOVERY trial, with more than 11000 participants and more than 2000 deaths, had excellent power to detect modest treatment benefits; none were observed.

The RECOVERY trial only studied patients who had been hospitalised with COVID-19; therefore, we are not able to provide any evidence on the safety and efficacy of colchicine used in other patient groups. In the COLCORONA trial ${ }^{26}$ of 4488 non-hospitalised patients with laboratory confirmed or clinically suspected COVID-19, fewer patients in the colchicine group died or were hospitalised within 30 days of randomisation than in the placebo group. However, the trial was stopped before the scheduled sample size had been fully enrolled due to logistical reasons, and the result was not statistically significant (odds ratio 0 79 [95\% CI 0 - 61-1.03]; $\mathrm{p}=0 \cdot 081$ ). ${ }^{26}$ Thus, the role of colchicine in treatment of COVID-19 in patients not requiring hospitalsation remains uncertain. Future trials in this setting are ongoing. ${ }^{27}$

In summary, the results of this large, randomised trial do not support the use of colchicine in adults hospitalised with COVID-19.

\section{Contributors}

This manuscript was initially drafted by the PWH and MJL, developed by the Writing Committee, and approved by all members of the trial steering committee. PWH and MJL vouch for the data and analyses, and for the fidelity of this report to the study protocol and data analysis plan. PWH, JKB, MB, LCC, JD, SNF, TJ, EJ, KJ, WSL, AMo, AMu, KR, GT, MM, RH, and MJL designed the trial and study protocol. MM, MC, G P-A, LP, MW, LW, ST, BC, CW, CG, PH, BP, TG, and AW-the Data Linkage team at the RECOVERY Coordinating Centre-and the Health Records and Local Clinical Centre staff-listed in the appendix (pp 2-25) — collected the data. ES, NS, and JRE accessed and verified the data and did the statistical analysis. All authors contributed to data interpretation and critical review and revision of the manuscript. PWH and MJL had access to the study data and had final responsibility for the decision to submit for publication.

Writing Committee (on behalf of the RECOVERY Collaborative Group) Peter W Horby, Mark Campbell, Enti Spata, Jonathan R Emberson, Natalie Staplin, Guilherme Pessoa-Amorim, Leon Peto, Martin Wiselka, Laura Wiffen, Simon Tiberi, Ben Caplin, Caroline Wroe,

Christopher Green, Paul Hine, Benjamin Prudon, Tina George, Andrew Wight, J Kenneth Baillie, Buddha Basnyat, Maya Buch, Lucy C Chappell, Jeremy Day, Saul N Faust, Raph L Hamers, Thomas Jaki, Edmund Juszczak, Katie Jeffery, Wei Shen Lim, Alan Montgomery, Andrew Mumford, Kathryn Rowan, Guy Thwaites, Marion Mafham, Richard Haynes, and Martin J Landray. Peter W Horby, Mark Campbell, and Enti Spata contributed equally. Marion Mafham, Richard Haynes, and Martin J Landray contributed equally.

\section{Data Monitoring Committee}

Peter Sandercock, Janet Darbyshire, David DeMets, Robert Fowler, David Lalloo, Mohammed Munavvar (from January 2021), and Ian Roberts (until December 2020), Janet Wittes.

\section{Declaration of interests}

We declare no competing interests.

\section{Data sharing}

The protocol, consent form, statistical analysis plan, definition and derivation of clinical characteristics and outcomes, training materials, regulatory documents, and other relevant study materials are available online. As described in the protocol (appendix 2), the trial Steering Committee will facilitate the use of the study data and approval will not be unreasonably withheld. Deidentified participant data will be made available to bona fide researchers registered with an appropriate institution within 3 months of publication. However, the Steering Committee will need to be satisfied that any proposed publication is of high quality, honours the commitments made to the study participants in the consent documentation and ethical approvals, and is compliant with relevant legal and regulatory requirements (eg, relating to data protection and privacy). The Steering Committee will have the right to review and comment on any draft manuscripts prior to publication. Data will be made available in line with the policy and procedures described online. Those wishing to request access should complete the online form and sent to data.access@ndph.ox.ac.uk

\section{Acknowledgments}

We would like to thank the thousands of patients who participated in this trial. We would also like to thank the many doctors, nurses, pharmacists, other allied health professionals, and research administrators at 177 NHS hospital organisations across the whole of the UK, supported by staff at the National Institute of Health Research (NIHR) Clinical Research Network, NHS DigiTrials, Public Health England, Department of Health \& Social Care, the Intensive Care National Audit \& Research Centre, Public Health Scotland, National Records Service of Scotland, the Secure Anonymised Information Linkage at University of Swansea, Swansea, UK, and the NHS in England, Scotland, Wales and Northern Ireland. The RECOVERY trial is supported by grants to the University of Oxford, Oxford, UK, from UK Research and Innovation and NIHR (MC_PC_19056), the Wellcome Trust (222406/Z/20/Z) through the COVID-19 Therapeutics Accelerator, and by core funding provided by the NIHR Oxford Biomedical Research Centre, Oxford, UK, the Wellcome Trust, the Bill and Melinda Gates Foundation, the Foreign, Commonwealth and Development Office, Health Data Research UK, the Medical Research Council Population Health Research Unit, the NIHR Health Protection Unit in Emerging and Zoonotic Infections, and NIHR Clinical Trials Unit Support Funding. TJ is supported by a grant from UK Medical Research Council (MC_UU_0002/14) and an NIHR Senior Research Fellowship (NIHR-SRF-2015-08-001). WSL is supported by core funding provided by NIHR Nottingham Biomedical Research Centre,

Nottingham, UK. Combiphar (Jakarta, Indonesia) supplied colchicine free of charge for use in this trial in Indonesia. Tocilizumab was provided free of charge for this trial by Roche (Basel, Switzerland). REGN-COV2 was provided free of charge for this trial by Regeneron (Tarrytown, NY, USA). Convalescent plasma was collected by NHS Blood and Transplant, the Scottish National Blood Transfusion Service, Welsh Blood Service, Northern Ireland Blood Transfusion Service and funded by the Department of Health and Social Care through core funding and funding under COVID-19 and EU SoHo Grant. The views expressed in this publication are those of the authors and not necessarily those of the NHS, the NIHR, or the UK Department of Health and Social Care.

\section{References}

1 Huang C, Wang Y, Li X, et al. Clinical features of patients infected with 2019 novel coronavirus in Wuhan, China. Lancet 2020; 395: 497-506.

2 Ruan Q, Yang K, Wang W, Jiang L, Song J. Clinical predictors of mortality due to COVID-19 based on an analysis of data of 150 patients from Wuhan, China. Intensive Care Med 2020; 46: 846-48.

3 Wang JH, Chen RD, Yang HK, et al. Inflammation-associated factors for predicting in-hospital mortality in patients with COVID-19. J Med Virol 2021; 93: 2908-2917.

4 Thwaites RS, Sanchez Sevilla Uruchurtu A, Siggins MK, et al Inflammatory profiles across the spectrum of disease reveal a distinct role for GM-CSF in severe COVID-19. Sci Immunol 2021; 6: eabg9873.

5 McElvaney OJ, McEvoy NL, McElvaney OF, et al. Characterization of the inflammatory response to severe COVID-19 illness. Am J Respir Crit Care Med 2020; 202: 812-21.

6 Dorward DA, Russell CD, Um IH, et al. Tissue-specific immunopathology in fatal COVID-19. Am J Respir Crit Care Med 2021; 203: 192-201.
For the online form see https:// www.ndph.ox.ac.uk/files/about/ data_access enquiry form_13_6_2019.docx 
7 RECOVERY Collaborative Group, Horby P, Lim WS, et al. Dexamethasone in hospitalized patients with Covid-19. N Engl J Med 2021; 384: 693-704.

8 Sterne JAC, Murthy S, Diaz JV, et al. Association between administration of systemic corticosteroids and mortality among critically ill patients with COVID-19: a meta-analysis. JAMA 2020; 324: $1330-41$.

9 RECOVERY Collaborative Group. Tocilizumab in patients admitted to hospital with COVID-19 (RECOVERY): a randomised, controlled, open-label, platform trial. Lancet 2021; 397: 1637-45

10 Kalil AC, Patterson TF, Mehta AK, et al. Baricitinib plus remdesivir for hospitalized adults with Covid-19. N Engl J Med 2020; 384: 795-807.

11 Broz P, Dixit VM. Inflammasomes: mechanism of assembly, regulation and signalling. Nat Rev Immunol 2016; 16: 407-20.

12 Rodrigues TS, de Sa KSG, Ishimoto AY, et al. Inflammasomes are activated in response to SARS-CoV-2 infection and are associated with COVID-19 severity in patients. J Exp Med 2021; 218: e20201707.

13 Dalbeth N, Lauterio TJ, Wolfe HR. Mechanism of action of colchicine in the treatment of gout. Clin Ther 2014; 36: 1465-79.

14 Fiolet ATL, Opstal TSJ, Mosterd A, et al. Efficacy and safety of lowdose colchicine in patients with coronary disease: a systematic review and meta-analysis of randomized trials. Eur Heart J 2021 42: 2765-775.

15 Martinez GJ, Celermajer DS, Patel S. The NLRP3 inflammasome and the emerging role of colchicine to inhibit atherosclerosisassociated inflammation. Atherosclerosis 2018; 269: 262-71.

16 Imazio M, Brucato A, Cemin R, et al. A randomized trial of colchicine for acute pericarditis. N Engl J Med 2013; 369: 1522-28.

17 Tardif JC, Kouz S, Waters DD, et al. Efficacy and safety of low-dose colchicine after myocardial infarction. N Engl J Med 2019; 381: 2497-505

18 Salehzadeh F, Pourfarzi F, Ataei S. The impact of colchicine on the COVID-19 patients; a clinical trial study. Research Square 2020; published online Sept 21. https://doi.org/10.21203/rs.3.rs-69374/v1 (preprint)
19 Lopes MI, Bonjorno LP, Giannini MC, et al. Beneficial effects of colchicine for moderate to severe COVID-19: a randomised, doubleblinded, placebo-controlled clinical trial. RMD Open 2021; 7: e001455.

20 Deftereos SG, Giannopoulos G, Vrachatis DA, et al. Effect of colchicine vs standard care on cardiac and inflammatory biomarkers and clinical outcomes in patients hospitalized with coronavirus disease 2019: the GRECCO-19 randomized clinical trial. JAMA Netw Open 2020; 3: e2013136.

21 RECOVERY Collaborative Group, Horby P, Mafham M, et al. Effect of hydroxychloroquine in hospitalized patients with Covid-19. N Engl J Med 2020; 383: 2030-40.

22 RECOVERY Collaborative Group. Lopinavir-ritonavir in patients admitted to hospital with COVID-19 (RECOVERY): a randomised, controlled, open-label, platform trial. Lancet 2020; 396: 1345-52.

23 RECOVERY Collaborative Group. Azithromycin in patients admitted to hospital with COVID-19 (RECOVERY): a randomised, controlled, open-label, platform trial. Lancet 2021; 397: 605-12.

24 RECOVERY Collaborative Group. Convalescent plasma in patients admitted to hospital with COVID-19 (RECOVERY): a randomised, controlled, open-label, platform trial. Lancet 2021; 397: 2049-59.

25 Deftereos SG, Siasos G, Giannopoulos G, et al. The Greek study in the effects of colchicine in COvid-19 complications prevention (GRECCO-19 study): rationale and study design. Hellenic J Cardiol 2020; 61: 42-45.

26 Tardif J-C, Bouabdallaoui N, L'Allier PL, et al. Colchicine for community-treated patients with COVID-19 (COLCORONA): a phase 3, randomised, double-blinded, adaptive, placebo-controlled, multicentre trial. Lancet Respir Med 2021; 9: 924-32.

27 University of Oxford. PRINCIPLE COVID-19 treatments trial widens to under 50s and adds colchicine. 2021. https://www. principletrial.org/news/principle-covid-19-treatments-trial-widensto-under-50s-adds-colchicine (accessed March 11, 2021). 